\title{
Symbole der Friedensbewegung
}

\section{Ein Blick auf ihre Plakate}

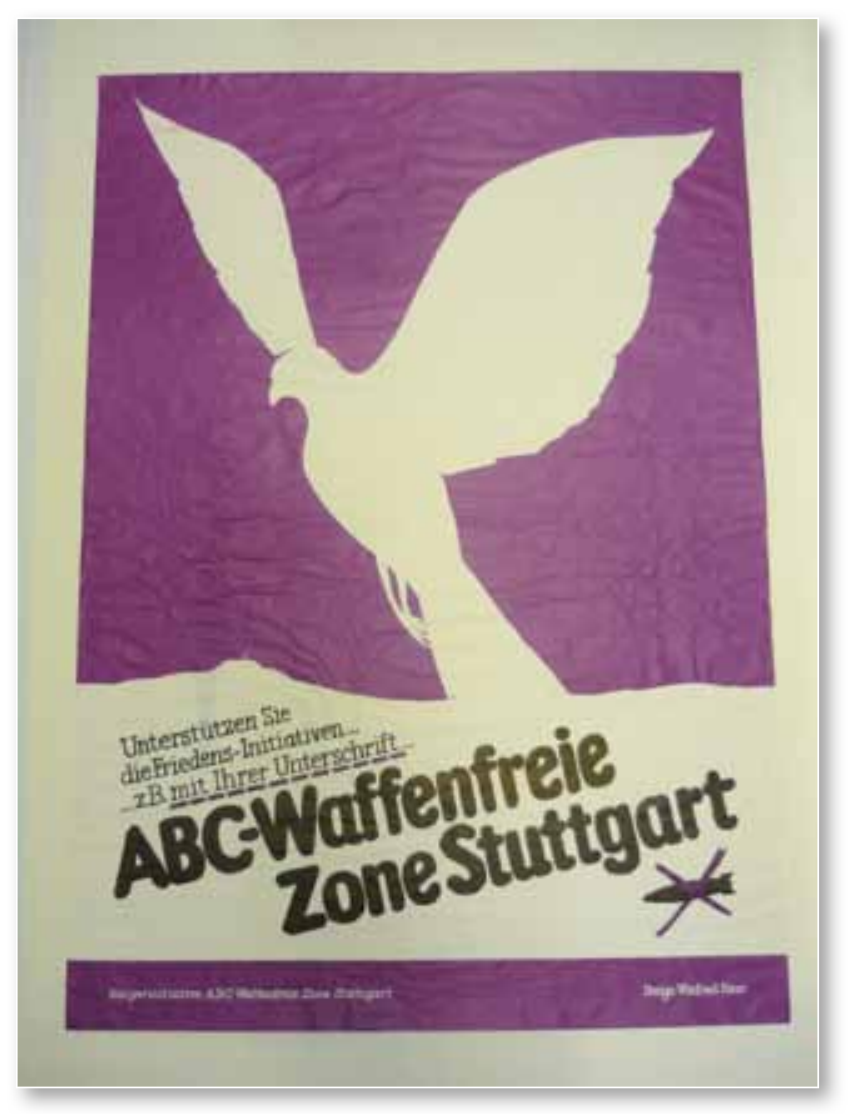

Abb. 1: Plakat der Bürgerinitiative "ABC-Waffenfreie Zone Stuttgart" von Winfried Baur, Stuttgart 1983, Signatur P 1050296

In der Sondersammlung "Neue Soziale Bewegungen" der Bibliothek für Zeitgeschichte befinden sich über 1.500 Plakate zum Thema Friedensbewegung. Seit mehr als 50 Jahren gehen Menschen auf die Straße, um gegen Kriege, Aufrüstung und andere Missstände zu demonstrieren. Auch beteiligt sich die Friedensbewegung immer wieder an aktuellen politischen Diskussionen. Die Friedensbewegung ist einer der Sammelschwerpunkte der 1972 gegründeten „Dokumentationsstelle für unkonventionelle Literatur", wie sie damals noch hieß. Ein Teil der Plakate wurde digitalisiert und kann über die Plakat-

(1) http://avanti.wlb-stuttgart.de/bfz/plakat/

(2) Kamps, Johannes: Plakat, Tübingen 1999, S. 38

(3) Ebda., S. 39f. sowie Deiters, Franz Josef: Bilder ohne Rahmen. Zur Rhetorik des Plakats, in: Knape, Joachim (Hg.): Medienrhetorik, Tübingen 2005, S. 81-112, hier S. $82 f$.

(4) Dorn, Margit: Plakat, in: Hügel, Hans-Otto (Hg.): Handbuch Populäre Kultur. Begriffe, Theorien und Diskussionen, Stuttgart 2003, S. 339342, hier S. 339.

(5) Diederich, Reiner: "Ohne kleine Leute keine großen Kriege". Zur Geschichte des Friedensplakates, in: Ders. (Hg.): Plakate gegen den Krieg: Dokumente der internationalen Friedensbewegung seit 1912, Weinheim 1983, S. [1]-[15], hier S. [11].
Datenbank der Bibliothek für Zeitgeschichte ${ }^{1}$ online eingesehen werden.

Plakate der Friedensbewegung gehören zu den am meisten nachgefragten Beständen der Sammlung. Nichtsdestotrotz bieten Plakate allgemein und insbesondere die Plakate der Friedensbewegung noch viel Stoff für studentische und wissenschaftliche Arbeiten, denn hier existieren noch einige Forschungslücken. ${ }^{2}$ Gleichzeitig sind Plakate, wie im Folgenden gezeigt werden wird, immer auch ein Spiegel ihrer Zeit, in denen der jeweilige Zeitgeist zum Ausdruck kommt. Schlagworte, unter denen Material zur Friedensbewegung zu finden ist, sind die Anti-Atomkraft-Bewegung, Kirche und Friedensbewegung oder Grüne und Friedensbewegung. Auch die Themen Nuklearwaffen, Eurofighter, Kriegsspielzeug, Frauen und Militär oder Bundeswehr-Auslandseinsätze stehen in Zusammenhang mit der Friedensbewegung.

Plakate dienen der Werbung, sei es für ein Produkt, für eine Partei oder für den Frieden. Das Plakat ist vorwiegend ein Medium der Straße und der Stadt, des urbanen Raums. ${ }^{3}$ Die Wirkungsmechanismen von Plakaten sind an den Augenblick gebunden.

Margit Dorn schildert dies wie folgt: „Der Kommunikationsprozess ist entsprechend kurz, flüchtig, häufig sogar unbewusst und auf eine zentrale Botschaft beschränkt". ${ }^{4}$

Trotz unterschiedlichster Konzeptionen, Technik und künstlerischer Handschrift bleibt das Repertoire der Symbole und Bildideen auf Plakaten begrenzt. Das hängt damit zusammen, dass die Sprache des Plakates im Gegensatz zu anderen Bereichen der Bildenden Kunst auf relativ eindeutige Zeichen angewiesen ist, die der Betrachter mit einem Blick erfassen muss. Der kubanische Grafiker Félix Béltran meint dazu: „Auf dem Plakat kann ein Symbol ,mehr' mit ,weniger' sagen, wenn es das Überflüssige weglässt und ein Minimum an präziser Information bietet, um die Aussage verständlich zu machen. Es hat gerade deshalb mehr Anziehungskraft, weil es eine visuelle Synthese erforderlich macht ". ${ }^{5}$ Hieraus erklärt sich die Vorliebe für einige wenige Symbole, 
die immer wiederkehren. Während Historiker vor einigen Jahren noch darauf hinwiesen, dass Plakate allein keine Hauptquelle historischer Erkenntnis seien, wird in der neueren Geschichtsforschung den Plakaten eine ähnlich wichtige Bedeutung beigemessen wie Texten. ${ }^{6}$ Anhand von ausgewählten Beispielen sollen im Folgenden drei zentrale Symbole der Friedensbewegung näher betrachtet werden: die Taube, Ruinen und (Atom-)Bomben.

\section{Die Taube als Friedenssymbol}

Das sicher bekannteste und am weitesten verbreitete Symbol der Friedensbewegung ist die Taube. Sie wurde im Laufe der Jahrhunderte in unterschiedlichsten Zusammenhängen und Variationen als Symbol verwendet und bisweilen auch missbraucht. ${ }^{7}$ Die wohl berühmteste Friedenstaube geht auf den Maler Pablo Picasso zurück, ${ }^{8}$ doch die Verwendung der Taube als Friedenssymbol reicht zurück bis ins Alte Ägypten. Im Laufe der Zeit war die Taube unter anderem Sinnbild für Friedfertigkeit, Unschuld, Frömmigkeit, Einfalt, weibliche Keuschheit und Kinderliebe. ${ }^{9}$ Auch in der christlichen Ikonographie kommt die Taube sehr oft vor. So erscheint sie unter anderem im ersten Buch Mose (Mos. 1.8, 10), das vom Ende der Sintflut berichtet, als Friedenssymbol. Sie bekundet hier den Frieden, den Gott mit den Menschen geschlossen hat.

Die Taube ist allerdings auch für kriegerische Zwecke instrumentalisiert worden. In beiden Weltkriegen fand sie als Symbol auf Kriegsanleihe-Plakaten Verwendung - sie sollte helfen, den Krieg zu finanzieren. ${ }^{10}$ Mit dem Aufkommen von Brieftauben Ende des 19. Jahrhunderts nahmen die Vögel sogar als lebende Tiere am Krieg teil: Im Ersten und auch noch im Zweiten Weltkrieg dienten sie als Nachrichtenübermittler an der Front. Später beabsichtigte man - falls ein dritter Weltkrieg bevorstünde - die Tiere "als Träger biologischer Waffen einzusetzen", da sie "von Radargeräten nicht geortet werden können". ${ }^{11}$

Ein regionales Beispiel für die Verwendung der Friedenstaube durch die Friedensbewegung ist das Plakat der Bürgerinitiative "ABC Waffenfreie Zone Stuttgart" von Winfried Baur aus dem Jahr 1983 (Abb. 1). Das Plakat ruft die Betrachter dazu auf, z.B. mit ihrer Unterschrift die Friedensinitiative zu unterstützen. Unter dem schwarz auf weiß gedruckten Plakat-Titel "ABC-Waffenfreie Zone Stuttgart" ist eine kleine durchgestrichene Bombe zu sehen; in der oberen Bildhälfte ist eine flügelschlagende Taube zu finden. Das Plakat ist relativ einfach und schlicht gehalten und zielt ganz auf die Wirkung der stilisierten Taube als bekanntes Symbol ab. Der Titel „ABC-Waffenfreie Zone Stuttgart" weist sowohl auf die örtliche als auch auf die grenzüberschreitende Dimension der Friedensbewegung hin. Global war die Forderung nach Abschaffung der nuklearen Rüstung und der Überwindung des Ost-West-Konflikts; lokal der Hinweis auf die Gefahr vor der eigenen Haustür. ${ }^{12}$

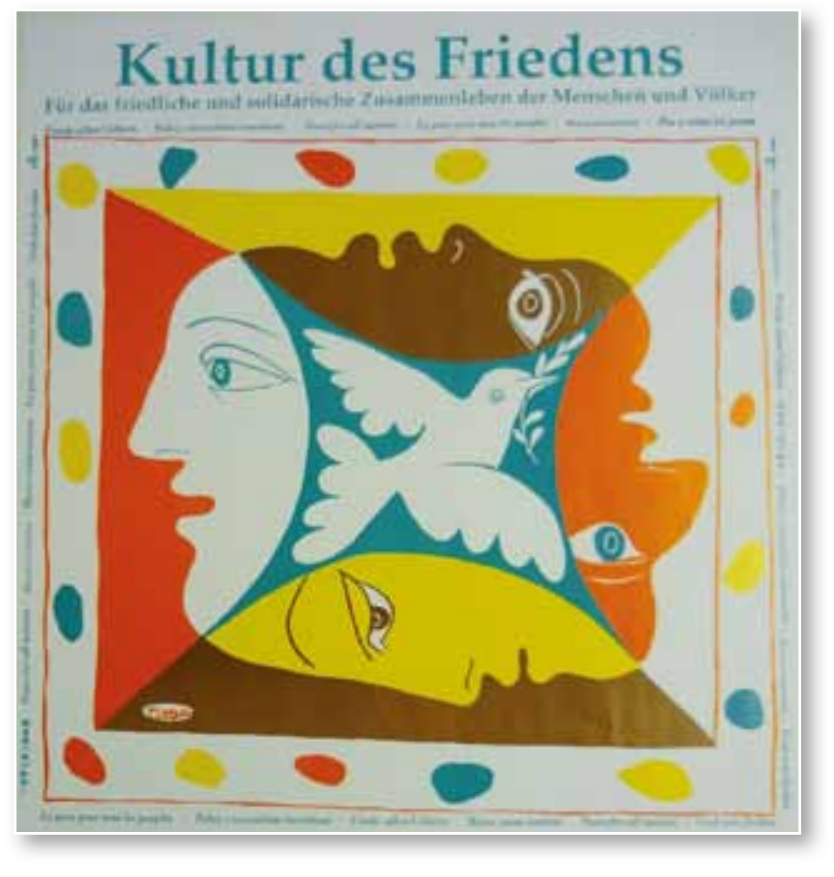

Abb. 2: Gesellschaft Kultur des Friedens (Hg.): Kultur des Friedens, Tübingen 2013, Signatur P 1050552

Ein weiteres Plakat aus dem vielfältigen Fundus friedensbewegter Anschläge ist überschrieben mit dem Titel: „Kultur des Friedens: Für das friedliche und solidarische Zusammenleben der Menschen und Völker" (Abb. 2). Es wurde 2013 von der "Gesellschaft Kultur des Friedens" zum 68. Jahrestag des

(6) Hagen, Manfred: Das politische Plakat als zeitgeschichtliche Quelle, in: Geschichte und Gesellschaft 4(1978), S. 412-436, hier S. 414; vgl. gegenwärtig: Paul, Gerhard: BilderMACHT. Studien zur Visual History des 20. und 21. Jahrhunderts, Göttingen 2013.

(7) Siehe besonders den voluminösen Bildband Baur, Ruedi u.a. (Hg.): Signs for Peace, Zürich 2013, insb. S. 20.

(8) Zur Friedenstaube bei Picasso siehe Kaulbach, Hans-Martin: Picasso und die Friedenstaube, in: Georges-Bloch-Jahrbuch des Kunstgeschichtlichen Seminars der Universität Zürich 4 (1997), S. 165-197.

(9) Pätzold, Kurt: Fliege über Berg und Tal. Friedenssymbol, Bibeltier, Nachrichtenbote und Kriegsteilnehmer: Die Taube im Wandel der Zeit, in: Junge Welt, Nr. 298, 2009, S. 4-5, hier S. 4.

(10) Pätzold: Fliege über Berg und Tal, S. 5.

(11) Ebd., S. 5.

(12) Kreis, Reinhild: Rüstungspolitik und Friedensbewegung. Flugblätter und Protestplakate der 1980er Jahre, in: Wobring, Michael (Hg.): Flugblätter - Plakate - Propaganda. Die Arbeit mit appellativen BildText-Dokumenten im Geschichtsunterricht, St. Ingbert 2013, S.7384, hier S. 77 
Endes des Zweiten Weltkriegs und zum Jubiläum "25 Jahre Kultur des Friedens" in Tübingen herausgebracht. ${ }^{13}$ Es zeigt farbenfroh die verschiedenen Gesichter der Friedensbewegung. Der Aufruf "Friede allen Völkern" richtet sich in vielen Sprachen an die Weltgemeinschaft und umrandet das Plakat. In der Mitte, umrahmt von den vier Gesichtern, ist eine Taube zu sehen, die in ihrem Schnabel einen Olivenzweig hält. Das Motiv stammt von Pablo Picasso, der es für das Weltjugendfestival 1951 in Berlin entworfen hat. Mit der Verwendung dieser Vorlage bedient sich die Friedensbewegung der Formensprache der modernen Kunst, die bereits selbst ikonenhafte Symbole zur Friedensbewegung hervorgebracht hat.

\section{Bomben und Ruinen}

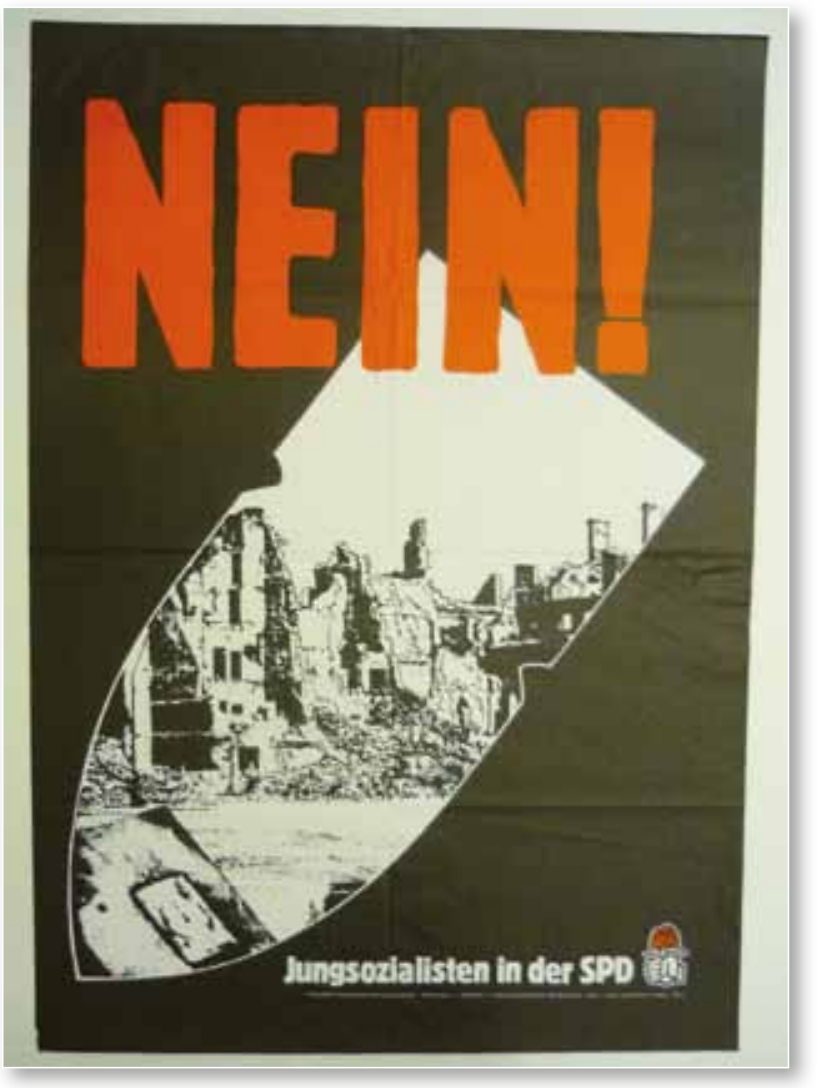

Abb. 3: Bundesvorstand der Jungsozialisten (Hg.): Nein!, Bonn, o.J., Signatur: $P 1050746$

Neben der Taube haben Bomben und Ruinen einen festen Platz in der Formensprache der Friedensbewegung (Abb. 3). Das Plakat stammt vom Bundesvorstand der Jungsozialisten in der SPD. Die Darstellungsmittel sind reduziert, die Farbpalette übersichtlich: drei Farben - rot, schwarz und weiß. Die Mittel, mit denen das Plakat arbeitet, prägen

(13) http://www.kulturdesfriedens.de/2013/05/25-jahre-kultur-des-friedens-2/ (14) Lenica, Jan: Moderne Plakatkunst von Trepkowski, Warschau 1958; Margadant, Bruno: Hoffnung und Widerstand. Das 20. Jahrhundert im Plakat der internationalen Arbeiter- und Friedensbewegung, Zürich 1998, S. 270; The Museum of Modern Art, Tadeusz Trepkowski: NIE!, http:// www.moma.org/collection/object.php?object_id=7792, Stand: 28.1.2015. sich sehr schnell ein: eine verwüstete Trümmerlandschaft im Umriss einer Bombe sowie das in roter Farbe hervorstechende "Nein!“ Die Ruinen verweisen auf die Wirkung der Bomben während des Zweiten Weltkriegs. Das Motiv nimmt Bezug auf ein

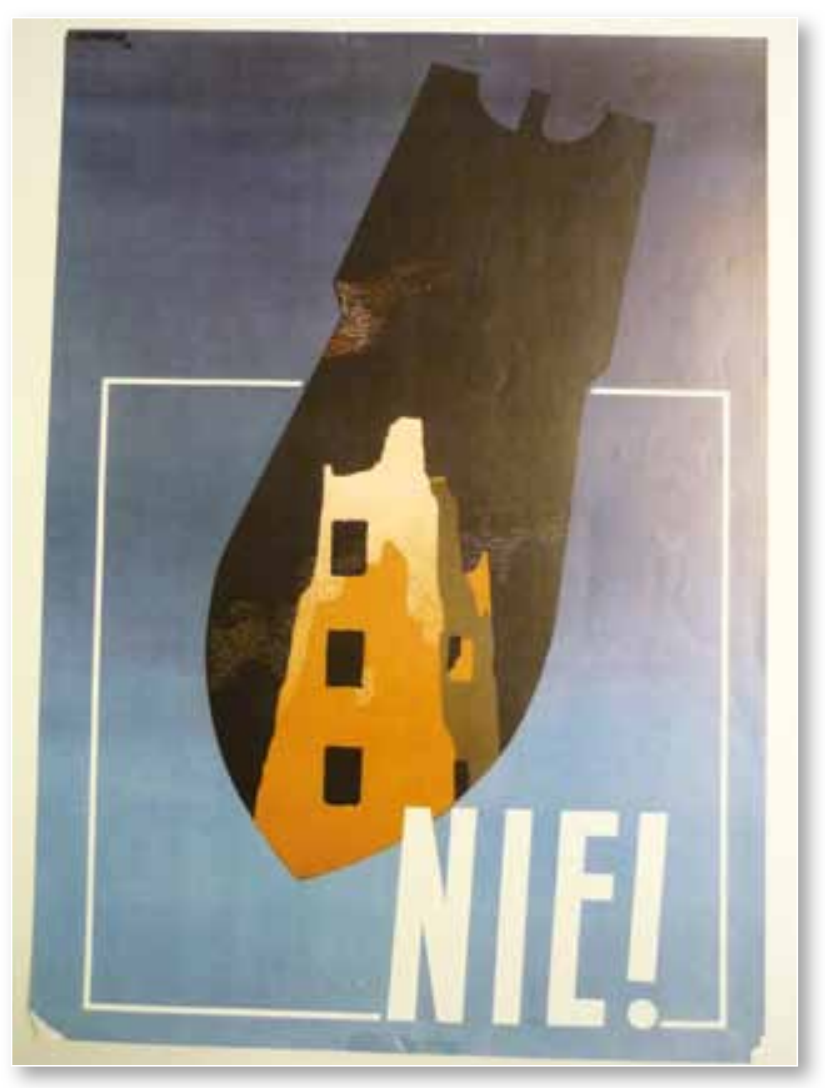

Abb. 4: Tadeusz Trepkowski: NIE!, o.O. 1952, Signatur: P 1050548

Plakat aus dem Jahre 1952 von Tadeusz Trepkowski, einem Altmeister der polnischen Plakatkunst (Abb. 4). Dieses Plakat ist ebenfalls recht minimalistisch gestaltet. Wie das Juso-Plakat zeigt es eine Ruine im Umriss einer Bombe sowie das polnische Wort für nein, "Nie!". Während der Hintergrund auf Abbildung 3 schwarz ist, wird er hier als hellblauer Himmel dargestellt. Das Hellblau wird allerdings nach oben hin immer dunkler, wodurch das Bild bedrohlich und düster wirkt. Die Bombe ist steiler ausgerichtet und scheint auf die Erde zuzurasen. Bild und Text stehen gleichgewichtig nebeneinander. Auf Friedensplakaten der unmittelbaren Nachkriegszeit weisen Ruinen oft auf die im Krieg systematisch zerstörten Städte hin und sind als Mahnung gedacht. Dies war naheliegend, denn die Ruinenlandschaften, die der Zweite Weltkrieg hinterlassen hatte, waren in dieser Zeit noch sehr präsent. Insbesondere Polen hatte unter dem Krieg zu leiden; so ist es wenig verwunderlich, dass ein polnischer Künstler auf das Motiv der Ruinen zurückgreift. ${ }^{14}$ 
Es war jedoch schwierig, mit den immer gleichen Symbolen noch eine Wirkung beim Betrachter zu erzielen. Mit der Kombination von Ruine und Bombe schuf Trepkowski ein neues, einprägsames Motiv. Da die Sammlung Neue Soziale Bewegungen erst 1972 gegründet wurde, sind Plakate aus der unmittelbaren Nachkriegszeit kaum vertreten. Das Bild der Ruinenlandschaft, wie sie der Zweite Weltkrieg hinterlassen hatte, prägte sich jedoch so stark im kollektiven Gedächtnis ein, dass noch Jahre später auf dieses Motiv zurückgegriffen wurde, wie Abbildung 3 zeigt. Es wirkt selbst bei Betrachtern, die den Krieg und die Ruinen der Nachkriegszeit nicht mehr selbst erlebt haben. Die Bombe wiederum als unmittelbares Symbol der Zerstörung blieb in ihren verschiedensten Ausprägungen immer ein wichtiges Symbol der Friedensbewegung.

\section{Der Atombombenpilz}

Die Bombe mit der größten Vernichtungskraft ist bis heute die Atombombe. Entsprechend hat auch sie Eingang gefunden in das Bildrepertoire der Friedensbewegung. Das Ende des Zweiten Weltkrieges, von Millionen Menschen als Befreiung empfunden, war überschattet vom Abwurf der ersten Atombomben auf die japanischen Städte Hiroshima und Nagasaki. Diese völlig neue Kriegführung, der Schock des Atomblitzes, der rasend schnelle Tod für Hunderttausende und die fürchterlichen Folgen für die Überlebenden förderten das Entstehen einer internationalen Friedensbewegung nach $1945 .^{15}$

Das hier vorgestellte Plakat (Abb. 5) aus dem Jahr 1982 versucht die Situation nach einem Atombombenabwurf zu veranschaulichen. Fast drei Viertel seiner Fläche wird von einer atomaren, milchig-gelben Wolke ausgefüllt. Der Titel "Ärzte warnen vor dem Atomkrieg. 2. Medizinischer Kongress zur Verhinderung eines Atomkrieges" weist sogleich im Untertitel darauf hin, dass man "Im Ernstfall - hilflos" ist. Ein Arzt liegt mit schmerzverzerrtem Gesicht auf dem Rücken, die Gliedmaßen teilweise verbrannt. Der neben inm liegende Erste-Hilfe-Koffer ist aufgebrochen und die Arztutensilien liegen verstreut herum. Im Hintergrund sieht man die zerstörte Gedächtniskirche in Berlin. Die Landschaft gleicht einem Trümmerfeld, man schaut in ein Inferno. Auch hier wird auf eine Ikone für die Zerstörungen des Zweiten Weltkriegs zurückgegriffen, obwohl die Gedächtniskirche nicht durch Nuklearwaffen zerstört wurde.

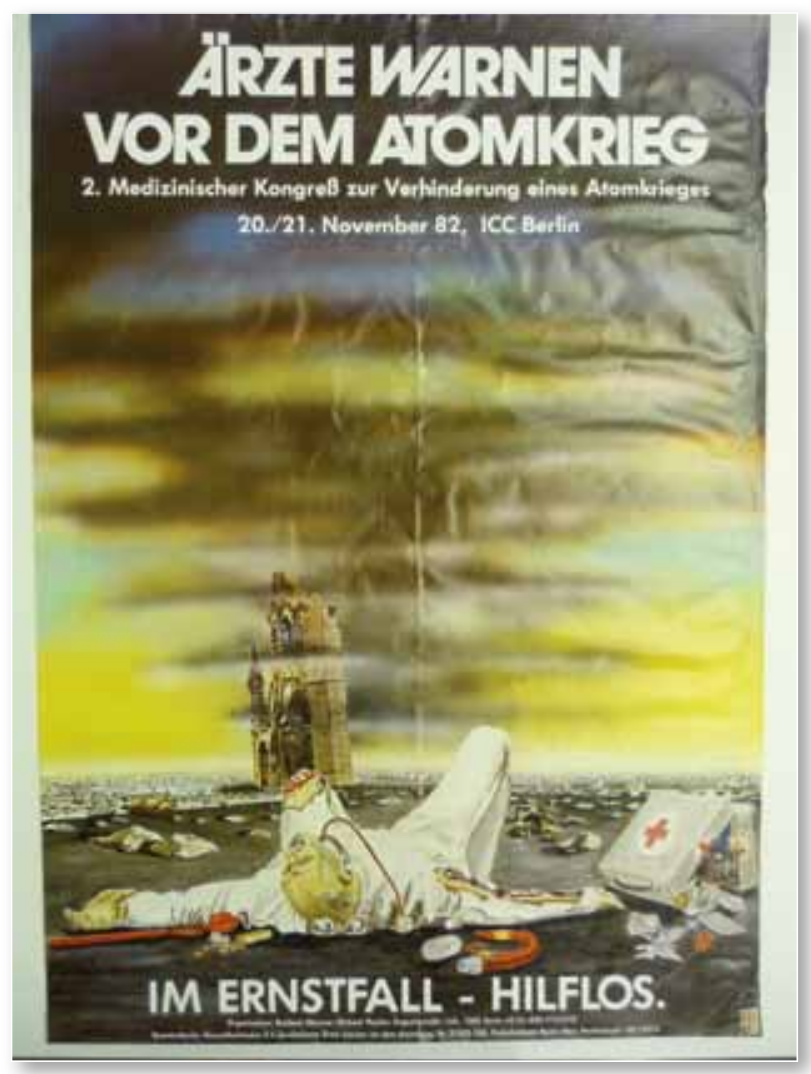

Abb. 5: Barbara Hövener u.a. (Hg.): Ärzte warnen vor dem Atomkrieg, Berlin (West) 1982, Signatur: P 1060260

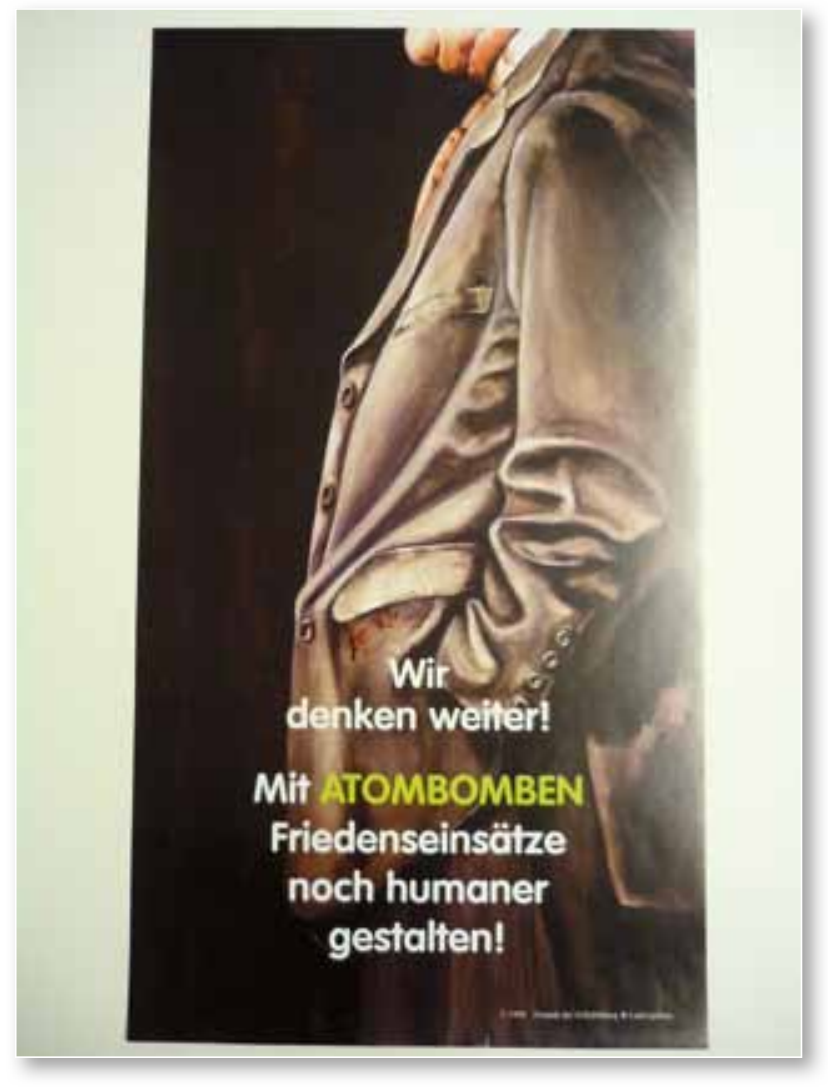

Abb. 6: Freunde der Volksbildung Ludwigsburg (Hg.): Wir denken weiter!, Ludwigsburg 1999, Signatur: P 1050534 
Mit ganz anderen Mitteln nähert sich dieses Plakat (Abb. 6) dem Thema Atomwaffen. Hier ist kein Atombombenpilz zu sehen. Stattdessen steht ein Mann, es könnte ein Manager oder ein Politiker sein, mit den Händen in der Hosentasche da; sein Gesicht ist nicht zu sehen. Dieses von den „Freunden der Volksbildung" aus Ludwigsburg 1999 geschaffene Plakat trägt den Titel: „Wir denken weiter! Mit Atombomben Friedenseinsätze noch humaner gestalten!“ Das Wort Atombomben ist neon-grün unterlegt. Die absurden Worthülsen lassen den Betrachter zunächst sprachlos zurück. Dadurch bleibt das Plakat im Gedächtnis, ohne dass auf altbekannte, vielleicht sogar abgegriffene Symbole zurückgegriffen werden muss.

\section{Resümee}

Ein Blick auf die Symbole Taube, Ruinen und (Atom-) Bomben zeigt, dass die Plakate der Sammlung Neue Soziale Bewegungen viele interessante Informationen für die historische und kulturwissenschaftliche Forschung enthalten. Gleichzeitig wird deutlich, dass die wissenschaftliche Auseinandersetzung mit Bildmotiven der Friedensbewegung noch in den Anfängen steckt. Bisherige Annahmen bedürfen der Überprüfung und für viele Fragen liegt bisher keine zufriedenstellende Antwort vor. So nehmen z.B. Plakate mit dem Symbol der Taube, entgegen anderslautender Auffassungen in der Forschung, durchaus Bezug auf die moderne Kunst. ${ }^{16}$

Die Verwendung von Ruinenlandschaften als Motiv Jahrzehnte nach dem Zweiten Weltkrieg deutet darauf hin, dass dieses Symbol ein fester Teil des kollektiven Bewusstseins geworden ist, auch wenn jüngere Generationen den Krieg und die durch inn entstandenen Ruinenlandschaften nicht mehr aus eigener Erfahrung gekannt haben.

Am Beispiel der Atombombe lässt sich hingegen beobachten, dass bestimmte Motive offenbar historisch geworden, sprich "aus der Mode gekommen" sind. In der Nachkriegszeit viel verwendet, zeigen neuere Plakate, dass der ikonische Atompilz nicht mehr unbedingt abgebildet wird, wenn von Nuklearwaffen die Rede ist. Die Erinnerung an Hiroshima und Nagasaki sowie die offenen Atomwaffentests der 1950er Jahre waren während des Kalten Krieges noch sehr präsent. Betrachter, die die heiße Phase des Kalten Krieges nicht mehr miterlebt haben, empfinden unter Umständen nicht dieselbe Betroffenheit. Bilder von Atombombenexplosionen sind heute wesentlich weniger in den Medien präsent. Sie erscheinen deswegen als eine weit entfernte, nicht akute Bedrohung. Es ist gut möglich, dass die zynische und aberwitzige Aussage von Abbildung 6 eher im Gedächtnis bleibt. Dies zu analysieren bleibt jedoch Aufgabe der Forschung, für die die Plakatsammlung der Bibliothek für Zeitgeschichte eine wichtige Fundgrube darstellt.

Michael Rost 